\title{
Self-Sealing of Nanoporous Low Dielectric Constant Patterns Fabricated by Nanoimprint Lithography**
}

\author{
By Hyun Wook Ro, Huagen Peng, Ken-ichi Niihara, Hae-Jeong Lee, Eric K. Lin, \\ Alamgir Karim, David W. Gidley, Hiroshi Jinnai, Do Y. Yoon, and Christopher L. Soles*
}

Highly porous nanoscale structures are critical for a range of emerging technologies. Prominent examples include semiconductor devices, ${ }^{[1-6]}$ sensors, ${ }^{[7]}$ separations, ${ }^{[8]}$ supports for catalyst ${ }^{[9]}$ and photonic devices. ${ }^{[10,11]}$ Recently, we demonstrated that it is feasible to directly pattern sub- $100 \mathrm{~nm}$ features with high fidelity into porous spin-on organosilicate glass (SOG) materials using nanoimprint lithography (NIL). ${ }^{[12]}$ This novel use of the NIL technology was motivated by the enormous cost savings that could be realized by directly patterning the interlayer dielectric (ILD) material suitable for semiconductor interconnect structures. Directly creating the pattern in the functional material eliminates the subsequent processing steps that are normally required to the transfer the sacrificial resist pattern into the functional material. ${ }^{[13,14]}$ This has the potential to greatly reduce the Back-End-Of-The-Line (BEOL) manufacturing costs. Some of these eliminated steps, such as the photoresist etching and ashing, are especially harmful to porous dielectrics with interconnected pore networks because the etching byproducts can diffuse into and contaminate the interior regions of the porous material. However, a new concern with the direct imprinting of the ILD material is that the patterns are essentially used "as-imprinted". If the imprinting process affects the key properties of the functional material, the performance of the

[*] Dr. C. L. Soles, Dr. H. W. Ro, Dr. H.-J. Lee, Dr. E. K. Lin, Dr. A. Karim NIST Polymers Division

100 Bureau Drive, stop 8541, Gaithersburg, MD 20899 (USA)

E-mail: csoles@nist.gov

Dr. H. Peng, Prof. D. W. Gidley

Department of Physics, University of Michigan

Ann Arbor, MI 48109 (USA)

K.-i Niihara, Prof. H. Jinnai

Department of Polymer Science and Engineering

Kyoto Institute of Technology

Kyoto 606-8585 (Japan)

Prof. D. Y. Yoon

Department of Chemistry, Seoul National University Seoul 151-747 (Korea)

[**] Official contribution of the National Institute of Standards and Technology; not subject to copyright in the United States. This work is supported in part by NIST Office of Microelectronic Program, University of Michigan, the System IC 2010 Project of Korea, and the Chemistry and Molecular Engineering Program of Brain Korea 21 Project. We also acknowledge the Nanofabrication Laboratory of the Center for Nanoscale Science and Technology (CNST) in NIST for providing facilities for the NIL process. resulting device can be impacted. This manuscript explores how NIL patterning affects the characteristics of the highly porous SOG patterns that are critical to their performance for ILD applications.

There are several technical and measurement challenges for highly porous SOG patterns. The porogen is typically an organic component that phase separates from the SOG during the spin coating process into nanoscale domains. The porogens volatilize at the elevated temperatures where the SOG is vitrified into a cross-linked network, templating nanoscale pores inside a hard, vitreous SOG matrix. The primary concern with direct patterning is that the process exposes this two phase material to large shear deformation flow fields, greatly increasing the surface or interfacial area. Then the two related issues are 1) pattern shrinkage/collapse and, 2) imprint-induced changes to the intended porosity upon vitrification and porogen volatilization. We previously showed that pattern shrinkage is of minimal concern in sub- $100 \mathrm{~nm}$ features imprinted in a porous SOG material. ${ }^{[12]}$ The focus here is to quantify how both NIL and porogen removal affect the pore characteristics of this material that are critical for determining the dielectric performance.

The NIL patterning process is described elsewhere in greater detail. ${ }^{[12]}$ Briefly, the thermal embossing form of NIL was used to create patterns onto as-cast films of a poly(methylsilsesquioxane) (PMSQ)-based material blended with $20 \%$ by volume of a thermally degradable porogen (Tetronics 150R1, purchased from $\mathrm{BASF}^{[15]}$ ). The PMSQ matrix material used in this study is prepared from methyltrimethoxysilane, dimethoxydimethylsilane and 1,2-bis(triethoxysilyl)ethane with a 7:1:2 molar ratio. ${ }^{[16]}$ Fully vitrified films of this PMSQ-based resin (without porogen) exhibit an elastic modulus of $10.5 \mathrm{GPa}$ and a dielectric constant of 2.84 at $1 \mathrm{MHz}^{[5]}$ Specular X-ray reflectivity (SXR), critical dimension small angle X-ray scattering (CD-SAXS), and field emission scanning electron microscopy (FE-SEM) were used to quantify the pattern fidelity and vitrification induced shrinkage with sub-nm level precision. These earlier measurements show that the NIL can reproducibly create patterns with a high fidelity of pattern transfer, regardless of the existence of the porogen. Vitrification induced shrinkage was also precisely characterized, revealing a small amount of vertical shrinkage in the pattern height direction but negligible change of pattern width; the patterns maintain their fidelity/shape through the vitrification and the porogen burn-out. 
In the present study, depth-profiled PALS, SXR and X-ray porosimetry (XRP) suitable for characterizing patterned samples, beyond planar films, ${ }^{[17-22]}$ are used to quantify the critical physical properties of the porous low- $k$ patterns such as the average pore size, pore size distribution, interconnectivity and depth profile of the porosity.

PALS is a pore/void volume characterization technique performed in a vacuum whereby the shortening of the annihilation lifetime of positronium (Ps) localized inside a pore, due to collisions of the Ps with the pore walls, directly correlates with the pore size. ${ }^{[17-19]}$ The PALS technique is particularly suited for characterizing pores in the $(0.2$ to 20$) \mathrm{nm}$ range. In addition, the detection of Ps escaping from the film to annihilate in the vacuum chamber of the instrument can be used to quantify the interconnectivity of the pores. ${ }^{[20]}$ Figure 1a shows the PALS-deduced pore size distributions (PSDs) for vitrified samples of the porogen-free and porogen-loaded samples both with and without NIL patterning. The unpatterned samples correspond to planar films that have been directly vitrified without going through the imprint process. The porogen-free, unpatterned film (dotted grey line) displays a PSD typical of PMSQ-type materials: a bimodal distribution of closed (not interconnected) micropores with average pore diameters of approximately $0.5 \mathrm{~nm}$ and $1.1 \mathrm{~nm}$. It is understood that the hydrolytic condensation reaction of the silsesquioxane precursors, organic-trifuctionalsilanes, leads to both cage-type architectures as well as more open or relaxed ladder-type networks. ${ }^{[6]}$ The smaller pores correspond to the dimensions of intra-cage pores while the larger pores reflect the inter-cage domains in the ladder network. ${ }^{[23]}$ NIL patterning shifts both of these peaks to pore diameters that are approximately 10 to $12 \%$ larger, suggesting an increase in the microporosity. It appears that the NIL process enhances the intrinsic nanoscale porosity of the PMSQ matrix material relative to the non-patterned material. We suspect that this results from residual solvent being trapped by the imprint mold which is in conformal contact with the film and prevents evaporation, effectively acting as a "small molecule porogen". The cross-linking process is partially completed during the imprint meaning that the nanoscale phase separated network is already being established, even before the final vitrification. Future studies will address this issue as a means of enhancing the microporosity.

An additional population of porogen-induced mesopores are seen in the PSDs of Figure 1a in the pore diameter range of (1.5 to 2.5) $\mathrm{nm}$. Comparing the PSDs of the unpatterned porogenfree and porogen-loaded films (dotted curves in Fig. 1a), we estimate that these induced mesopores account for about $2 / 3$ of the total porosity. The more interesting observation is the effect of imprinting on the porogen-loaded sample. Figure 1a shows a significant decrease in the population of mesopores and an increase in the population of micropores relative to the unpatterned material. We are forced to conclude that full porosity was not achieved as a result of either incomplete pore formation or pore collapse during vitrification. However, from the applications point of view, this might be an attractive and unexpected result of the imprint process since smaller pores are
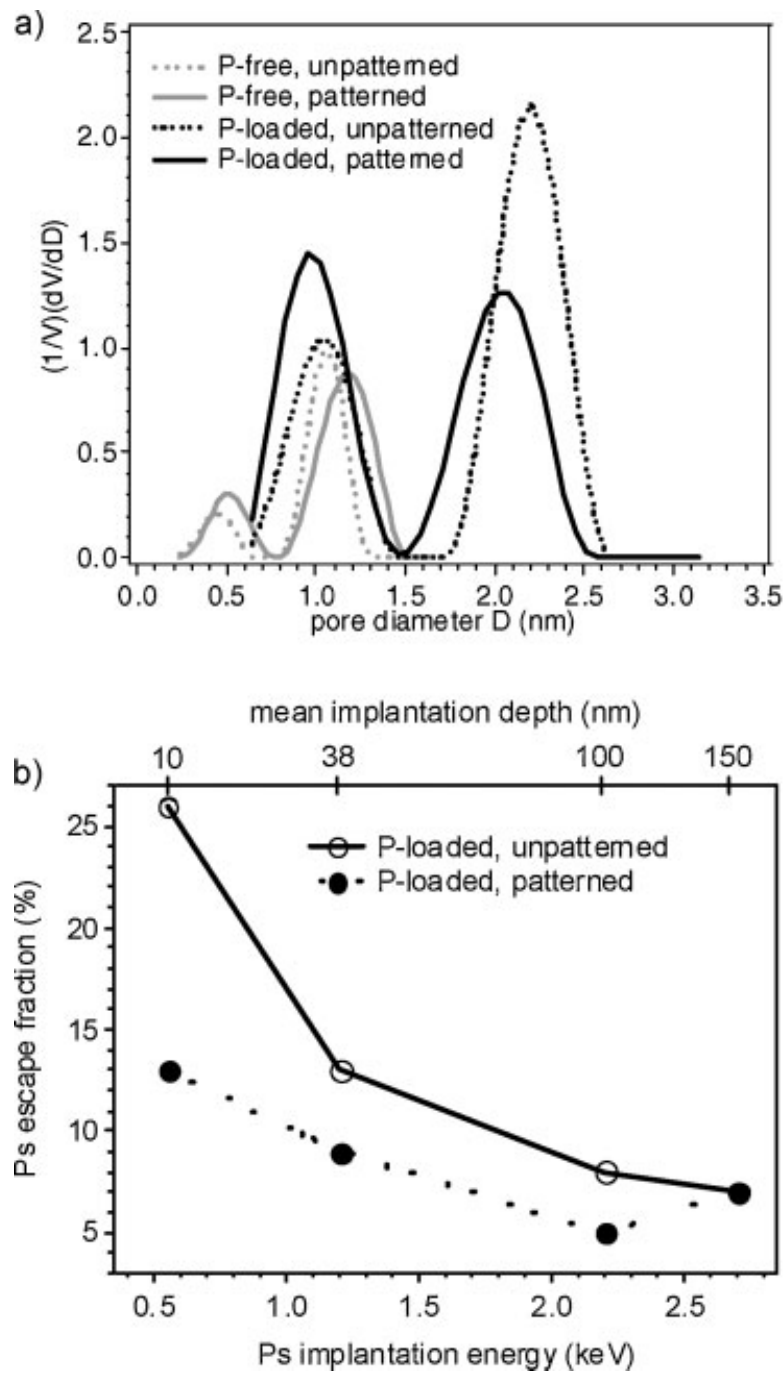

Figure 1. The pore size distributions of the four dielectric films fitted from the PALS spectra acquired at $2.7 \mathrm{keV}$ using the CONTIN program are shown in panel (a). The area under the PSD curve scales with the XRP measured porosity (will be shown later) of the entire film for unpatterned films and the residue layer for the patterned film. The Ps escape fraction, i.e., the relative amount of escaping vacuum Ps vs. the combined Ps intensity owing to porogen-induced pores, is plotted against positron implantation energy in panel (b). The Ps escape fraction typically increases with the decrease of beam energy, as Ps formed from shallowly implanted positrons are more prone to escape.

generally preferred for ultralow- $k(k<2.2)$ applications in order to minimize pore percolation or interconnection problems. The fact that imprinting decreases the mesopore $(>2 \mathrm{~nm})$ population and increases the micropore $(<2 \mathrm{~nm})$ population is a very attractive step in reaching this goal.

Figure 1b shows the Ps escape fraction for a few different Ps implantation depths, achieved by adjusting the acceleration voltage of the positron beam, in the porogen-loaded samples. The highest implantation energy corresponds to a depth where almost half of the positrons are penetrating into the supporting Si substrate. For the shallower implantation depths, where the 
positrons are completely stopped the SOG films, there is a significant reduction in the Ps escape fraction for the imprinted pattern. This is consistent with the reduced population of mesopores in Figure 1a resulting in a less interconnected pore network.

SXR measurements can also characterize porosity when it is combined with the mechanism of capillary condensation, in a technique that is referred to as $\mathrm{X}$ - ray porosimetry (XRP). The use of XRP to characterize critical porosity characteristics, such as total porosity, average film density, wall density between pores, pore size and the pore size distribution, has been well documented. ${ }^{[21,22]}$ Here, we extend the use of XRP from planar films to patterned structures through the effective medium approximation and a multi-layer modeling approach. The porous PMSQ films readily adsorb toluene with a negligible degree of swelling in a saturated toluene environment. Correspondingly, the difference in the scattering length density $\left(Q_{\mathrm{c}}{ }^{2}\right)$ between the sample in vacuum and in saturated toluene (for each layer in the model) is directly related with the amount of toluene absorbed in the pores. SXR measurements in vacuum give the average film density, $\rho_{\text {average }}$. The saturated film density, $\rho_{\text {saturated, }}$ can be provided from the SXR measurements under the presence of the vapor. The porosity, $P$, and wall density of the material between the intrinsic pores, $\rho_{\text {wall }}$, are determined by simultaneously solving the following equations:

$\rho_{\text {average }}=\rho_{\text {wall }} \times(1-P)$

$\rho_{\text {saturated }}=\rho_{\text {solvent }} \times P+\rho_{\text {wall }} \times(1-P)$

Figure 2a shows the SXR data for the vitrified samples both under vacuum (black lines) and in the presence of saturated toluene vapor (grey lines). In each case the $Q_{\mathrm{c}}$ for the Si does not change in the presence of toluene but the $Q_{\mathrm{c}} \mathrm{s}$ for the residual layer and patterned region of the PMSQ material shift to higher $Q$; the condensation of toluene inside of the pores in the pattern leads to increase of the total density. The increase in density is more pronounced for the porogenloaded sample and is consistent with the additional porosity generated by the porogen. However, even the porogen-free sample adsorbs some toluene, indicating a small amount of intrinsic porosity. Fits to the SXR data in Figure 2a are presented in Figure $2 \mathrm{~b}$ and $\mathrm{c}$ in terms of the $Q_{\mathrm{c}}{ }^{2}$ profiles as a function of distance $z$ through the pattern for the porogen-free and porogen-loaded samples. From these data and the equations above, we determine the porosity in the residual layer of the patterned films to be $(11.4 \pm 1.0) \%^{[24]}$ and

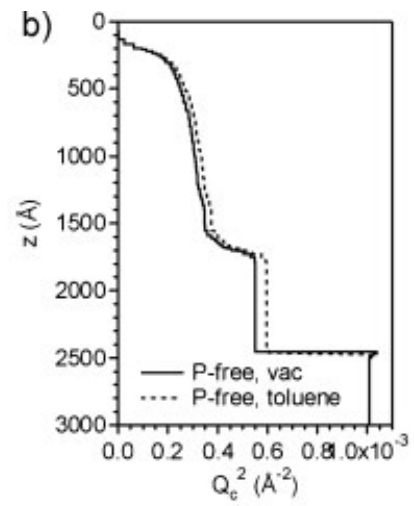

c)
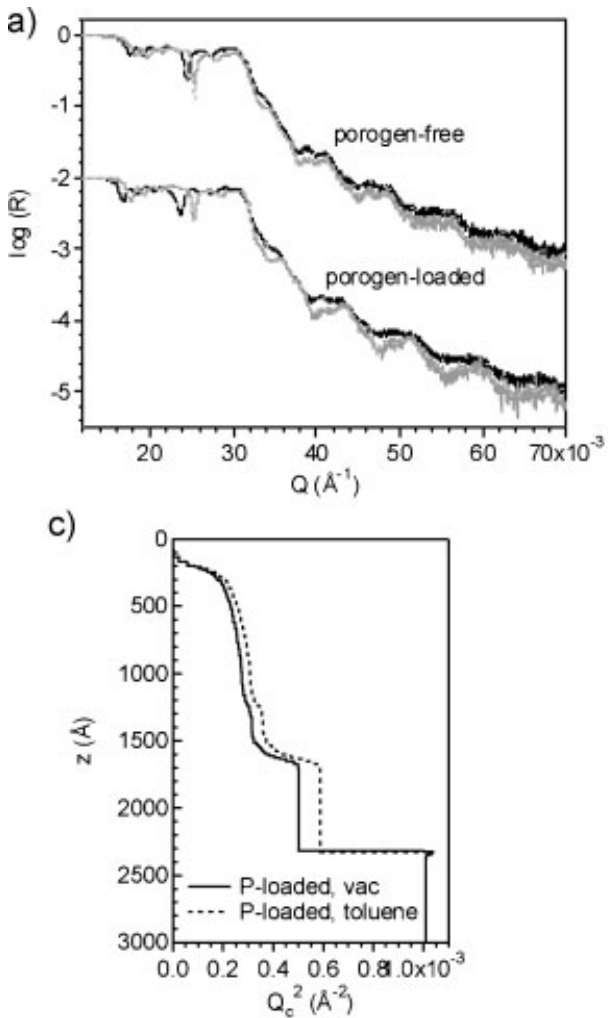

d)

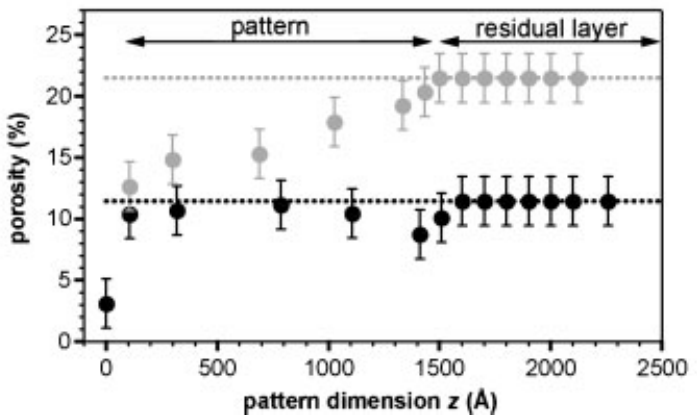

Figure 2. The reflectivity data and scattering length density profiles are shown for the samples before and after toluene absorption. Part a) shows the reflectivity of the porogen-free and porogen-loaded samples after vitrification both in vacuum (black lines) and in the presence of saturated toluene vapor (grey lines). Part b) and c) show how the scattering length density $\left(Q_{c}{ }^{2}\right)$ profiles change upon the adsorption of toluene into the pores for porogen-free and porogen-loaded patterns, respectively. d) Porosity profiles from the scattering length density changes in (b) and (c) are shown through both the residual layer and the patterned region. 
$(21.5 \pm 1.0) \%$ for the porogen-free and porogen-loaded samples, respectively. Unpatterned planar versions of these films (data not shown here) vitrified under identical conditions lead to porosities of $(8.5 \pm 1.0) \%$ and $(22.9 \pm 1.0) \%$, respectively. The residual layer of the patterned, porogenfree material has about $30 \%$ more intrinsic microporosity than the unpatterned analog, nominally consistent with the $\approx 10 \%$ increase in micropore diameter as detected by PALS. For the porogen-loaded sample, the patterned and nonpatterned residual layer porosities are within the experimental uncertainty.

There is also an increase in $Q_{\mathrm{c}}{ }^{2}$ in the patterned region upon the adsorption of toluene. The absolute magnitude of this shift in $Q_{\text {c }}{ }^{2}$ decreases with increasing height, approaching zero at the top of the patterns. As we have previously shown, the pattern widths decrease with height (the patterns are tapered) so a decrease in the absolute uptake with pattern height is reasonable. ${ }^{[12,25]}$ Thinner line widths mean less material and less adsorbed toluene on an absolute scale. In our previous publication on this topic, we demonstrated how the ratio of $Q_{\mathrm{c}}{ }^{2} \mathrm{~s}$ in the residual layer to pattern can be used, with an external calibration of pitch or line width, to determine the absolute line width variations as a function of height. ${ }^{[12,25]}$ If we know exactly how wide the pattern is at each height (and that the pattern does not swell with the toluene), a simple rule of mixtures can be used to convert the $Q_{\mathrm{c}}^{2}$ increase as a function of pattern height into porosity as a function of height. This is shown in Figure $2 \mathrm{~d}$ for both the porogen-free (black) and porogen-loaded patterns (grey). The horizontal dotted lines indicate the level of porosity in the residual layer. In the case of the porogen-free sample, the porosities in the patterned region and the residual layer are very similar. However, in case of the porogen-loaded sample, there is a decrease in the porosity with increasing pattern height. The porosity at the top of the pattern becomes the level of just the microporosity of the porogen-free material.

The origin for this decreased porosity near the tops of the pattern is not yet obvious. It is certainly consistent with the reduced mesoporosity detected by PALS, on average, in the patterned porous film. Immediately, two possible explanations are evident. The first is that the imprint process may result in a non-uniform distribution of porogen in the pattern. The fact that the porosity near the top of the pattern approaches the porosity of the porogen-free pattern is consistent with such a phase segregation picture. However, it may also be possible that the porogen remains well dispersed throughout the film during the imprint process but that the mesopores near the top and edges of the pattern preferentially collapse during the vitrification process. This would result in a dense skin around the porous pattern. Since the pattern is tapered from top to bottom, the dense skin would be a larger fraction of the total width at the top of the pattern and thus the decreasing porosity as a function of pattern height.

Cross-sectional transmission electron microscopy (TEM) images of the imprinted porous patterns were obtained to check the existence of a dense skin layer. This is a difficult task given that sectioning procedure (in this case focused ion beam milling) can induce artifacts in nanoporous low- $k$ materials. ${ }^{[26]}$ Nevertheless, great care was taken in preparing the sample cross-section to minimize the damage. The details of this sectioning process are described in the Experimental Section. Figure 3 shows the final cross-sectional TEM images, part (a) showing the electron energy loss images filtered for the $\mathrm{C}$ (left) and Si (right) signals and part (b) showing the total electron density contrast image. These images show that the pattern height of this line is approximately $140 \mathrm{~nm}$ while the residual layer thickness is approximately $60 \mathrm{~nm}$. These results are very consistent with the values of $142 \mathrm{~nm}$ and $67 \mathrm{~nm}$ for the pattern height and the residual layer, respectively, from the SXR data, suggesting that the damage minimization during the sectioning
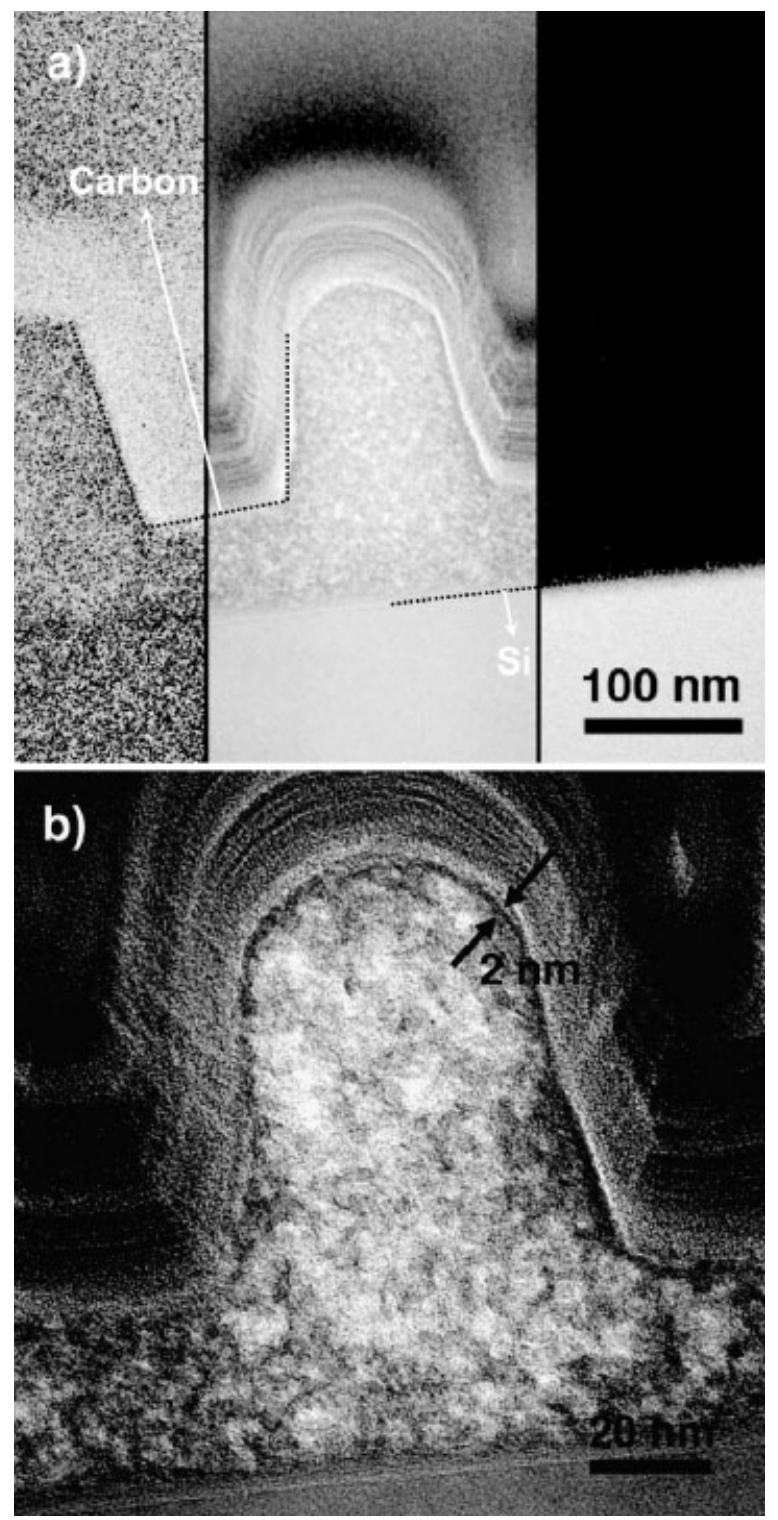

Figure 3. Cross-section TEM micrographs of porogen-loaded pattern. Part a) showing the electron energy loss images filtered for the $C$ (left) and Si (right) signals and part b) showing the total electron density contrast image. 
was successful. In part (a) of Figure 3, the highly striated structure conformal over the top of the pattern is the carbon coating. However, by carefully comparing the total electron density mapping image with the carbon mapping image in Figure 3a, especially in the continuity at the interface of the images, one sees signs of an additional layer that appears to be higher density (darker) at the surface of the pattern. As suggested by the arrows in Figure $3 b$, a dense skin of approximately $2 \mathrm{~nm}$ over the top of the patterns is reasonably consistent with the image. The strength of the TEM image alone in establishing the existence of such a dense skin on the surface of the pattern is tentative, if not speculative at best. However, in light of the supporting evidence provided by the XRP and PALS measurements and the general knowledge of the NIL process, a reduced porosity skin on the surface of the porous sample is a reasonable conclusion.

The combination of a vertical shrinkage upon vitrification (discussed in the previous publication), ${ }^{[12]}$ the reduced population of the porogen-induced mesopores, the attenuated Ps escape fraction, and the depth-dependent porosity would be consistent with a dense skin of non-mesoporous material at the surface of the pattern. One could conceive several different mechanisms by which a dense skin would arise at the surface of the porogen-loaded imprints. We have already alluded to a pore collapse mechanism. This would be reasonable at an interface where only biaxial stresses can exist; an inability to support a triaxial stress region to develop near the mold interface. In reality, the situation near an interface may be relevant to pore collapse. It should also be realized that the moderately hydrophilic PEO domains of the porogen are not compatible with the very hydrophobic nature of the fluorinated mold surface. During the imprint process there would be a thermodynamic driving force for a porogen-depleted is probably even more complex. In addition there will be large amounts of shearing during the imprint as the material slides past the static surfaces of the mold and into the cavities. These shear fields could alter the shape of the porogen domains and possibly even their phase behavior with the matrix material in a very non-trivial manner. At this time the exact origin of the dense skin is still unclear. However, it appears that the nanoporous low- $k$ pattern produced from NIL process induces a higher density skin layer at the boundary of the patterns which "protects" the nanoscale pores in the pattern. This generation of a dense skin layer around the nanoporous pattern yields an effect similar to the so-called pore sealing layer created by atomic layer deposition (ALD). ${ }^{[27,28]}$ Overall, the nanoporous low- $k$ patterns created from NIL in this study exhibit several promising aspects that are highly attractive from the practical point of view.

In conclusion, these results indicate that the imprint processes could have great potential for directly patterning and creating nanoporous low- $k$ patterns required for semiconductor interconnect fabrication. Nanoporous low- $k$ patterns are created by directly patterning onto as-cast films of a PMSQ-type SOG material with added porogen. This direct patterning process appears to tailor the nanoporous structures inside the patterns to the positive way. Moderate reductions of the porogen-induced mesoscale porosity are observed with a concomitant increase in the nanoscale intrinsic microporosity of the PMSQ-type material. The net effect is a decreased interconnectivity of the porous network, good for improving the barrier properties of the pattern. Perhaps more importantly, the reduced porosity seems to be selectively isolated to the surface of the pattern, effectively forming a dense skin. This dense skin layer may turn out to be beneficial given that in conventional lithography extra processes are often required to protect the porous low- $k$ patterns from reactive contamination sources during subsequent process steps. ${ }^{[29-31]}$ Direct imprinting may have the potential to create self-sealing, superior nanoporous low- $k$ patterns.

\section{Experimental}

NIL of PMSQs: Detailed information of NIL of PMSQs is described in the previous publication. [12]

Specular X-Ray Reflectivity (SXR): SXR measurements were performed on a Philips X'PERT diffractometer using $\mathrm{Cu} \mathrm{K} \alpha$ X-ray radiation $(\lambda=1.54 \AA)$. The incident beam is focused with a curved mirror into a 4-bounce Ge [220] crystal monochromator before being incident onto the sample. The reflected beam is further conditioned with a 3-bounce Ge [220] crystal monochromator ensure the specular condition. The angular reproducibility of the goniometers that control the sample rotation and angular position of X-ray detector was $0.0001^{\circ}$. The reflectivity was collected at $25^{\circ} \mathrm{C}$ under vacuum for all samples.

Positron Annihilation Lifetime Spectroscopy (PALS): PALS measurements were performed on the non-patterned version (planar films) of porous material in the vacuum chamber of an electrostatically-focused positron beam with ${ }^{22} \mathrm{Na}$ as the radioactive source of positrons. The positron beam energy was varied between ( 0.55 and 2.7$)$ $\mathrm{keV}$ to control the implantation depth of the positrons into the films. This energy range corresponds to a mean implantation depth of (10 to 150) $\mathrm{nm}$ based on a film density of $1.0 \mathrm{~g} \mathrm{~cm}^{-3}$. The lifetime spectra were fitted using both discrete and continuum fitting programs, i.e., POSFIT and CONTIN, to resolve positron/positronium lifetimes and intensities and pore size distribution (PSD).

Transmission Electron Microscopy (TEM): The thin sample was coated with carbon and subsequently coated with $\mathrm{Pt}$ in order to reduce charge and to enhance heat transfer during the ultra-thin sectioning using focused-ion-beam (FIB) (JEM-9310FIB, JEOL, Co., Ltd., Japan). The section was put on the $\mathrm{Cu}$ mesh grid for TEM experiments. The TEM observations were carried out on JEM-2200FS (JEOL, Co., Ltd., Japan) operated at $200 \mathrm{kV}$ equipped with a slow-scan CCD camera (Gatan USC1000, Gatan, Inc.) as a detector. Only the transmitted and elastically scattered electrons were selected by energy filter installed in JEM-2200FS (Omega filter, JEOL Co., Ltd., Japan).

Received: July 9, 2007 Revised: November 30, 2007 Published online: April 15, 2008

[1] C. V. Nguyen, K. R. Carter, C. J. Hawker, J. L. Hedrick, R. L. Jaffe, R. D. Miller, J. F. Remenar, H.-W. Rhee, P. M. Rice, M. F. Toney, M. Trollsas, D. Y. Yoon, Chem. Mater. 1999, 11, 3080.

[2] K. Landskron, B. D. Hatton, D. D. Perovic, G. A. Ozin, Science 2003, $302,266$. 
[3] R. A. Pai, R. Humayun, M. T. Schulberg, A. Sengupta, J.-N. Sun, J. J. Watkins, Science 2004, 303, 507.

[4] S. Yang, P. Mirau, C. S. Pai, O. Nalamasu, E. Reichmanis, J. C. Pai, S. Y. Obeng, J. Seputro, E. K. Lin, H.-J. Lee, J. Sun, D. W. Gidley, Chem. Mater. 2002, 14, 369.

[5] H. W. Ro, K. J. Kim, P. Theato, D. W. Gidley, D. Y. Yoon, Macromolecules 2005, 38, 1031 .

[6] H. W. Ro, K. Char, E.-c. Jeon, H.-J. Kim, D. Kwon, H.-J. Lee, J.-K. Lee, H.-W. Rhee, C. L. Soles, D. Y. Yoon, Adv. Mater. 2007, $19,705$.

[7] B. J. Scott, G. Wirnsberger, G. D. Stucky, Chem. Mater. 2001, 13, 3140.

[8] H. Dong, M. A. Brook, J. D. Brennan, Chem. Mater. 2005, 17, 2807.

[9] J. L. Defreese, A. Katz, Chem. Mater. 2005, 17, 6503.

[10] S. Y. Choi, M. Mamak, N. Coombs, N. Chopra, G. A. Ozin, Nano Lett. 2004, 4, 1231.

[11] M. C. Fuertes, F. J. Lopez-Alcaraz, M. C. Marchi, H. E. Troiani, V. Luca, H. Miguez, G. J. A. A. Soler-Illia, Adv. Funct. Mater. 2007, 17, 1247.

[12] H. W. Ro, R. L. Jones, H. Peng, D. R. Hines, H.-J. Lee, E. K. Lin, A. Karim, D. Y. Yoon, D. W. Gidley, C. L. Soles, Adv. Mater. 2007, 19, 2919.

[13] M. D. Stewart, C. G. Willson, MRS Bull. 2005, 30, 947.

[14] G. M. Schmid, M. D. Stewart, J. Wetzel, F. Palmieri, J. Hao, Y. Nishimura, K. Jen, E. K. Kim, D. J. Resnick, A. Liddle, C. G. Wilson, J. Vac. Sci. Technol. B 2006, 24, 1283.

[15] Certain commercial materials and equipment are identified in this paper in order to specify adequately the experimental procedure. In no case does such identification imply recommendation by the National Institute of Standards and Technology nor does it imply that the material or equipment identified is necessarily the best available for this purpose.

[16] D. Y. Yoon, H. W. Ro, E. S. Park, J.-K. Lee, H.-J. Kim, K. Char, H.-W. Rhee, D. Kwon, D. W. Gidley, Mater. Res. Soc. Symp. Proc. 2003, 766, 241.
[17] D. W. Gidley, W. E. Frieze, T. L. Dull, J. Sun, A. F. Yee, C. V. Nguyen, D. Y. Yoon, Appl. Phys. Lett. 2000, 76, 1282.

[18] T. L. Dull, W. E. Frieze, D. W. Gidley, J. N. Sun, A. F. Yee, J. Phys. Chem. B 2001, 105, 4657.

[19] D. W. Gidley, H.-G. Peng, R. S. Vallery, Annu. Rev. Mater. Res. 2006, 36,49 .

[20] H.-G. Peng, R. S. Vallery, M. Liu, W. E. Frieze, J.-H. Yim, H.-D. Jeong, J. Kim, D. W. Gidley, Appl. Phys. Lett. 2005, 87, 161903.

[21] H. J. Lee, C. L. Soles, D. W. Liu, B. J. Bauer, W. L. Wu, J. Polym. Sci. Part B 2002, 40, 2170.

[22] H. J. Lee, C. L. Soles, D. W. Liu, B. J. Bauer, E. K. Lin, W. L. Wu, A Grill, J. Appl. Phys. 2004, 95, 2355.

[23] C. Zhang, F. Babonneau, C. Bonhomme, R. M. Laine, C. L. Soles, H A. Hristov, A. F. Yee, J. Am. Chem. Soc. 1998, 120, 8380.

[24] The error bars presented throughout this manuscript indicate the relative standard uncertainty of the measurement.

[25] H. J. Lee, H. W. Ro, C. L. Soles, R. L. Jones, E. K. Lin, W.-L. Wu, D R. Hines, J. Vac. Sci. Technol. B 2005, 23, 3023.

[26] L. E. Thompson, P. M. Rice, E. Delenia, V. Y. Lee, P. J. Brock, T. P. Magbitang, G. Dubois, W. Volksen, R. D. Miller, H.-C. Kim, Microsc. Microanal. 2006, 12, 156.

[27] Y. Travaly, J. Schuhmacher, M. R. Baklanov, S. Giangrandi, O. Richard, B. Brijs, M. Van Hove, K. Maex, T. Abell, K. R. F. Somers, M. F. A. Hendrickx, L. G. Vanquickenborne, A. Ceulemans, A. M. Jonas, J. Appl. Phys. 2005, 98, 083515.

[28] Y.-B. Jiang, N. Liu, H. Gerung, J. L. Cecchi, C. J. Brinker, J. Am. Chem. Soc. 2006, 128, 11018.

[29] S.-S. Hwang, H.-C. Lee, H. W. Ro, D. Y. Yoon, Y.-C. Joo, Appl. Phys. Lett. 2005, 87, 11915 .

[30] Z. Chen, K. Prasad, N. Jiang, L. J. Tang, P. W. Lu, C. Y. Li, J. Vac. Sci. Technol. B 2005, 23, 1866.

[31] X. Hua, M.-S. Kuo, G. S. Oehrein, R. Lazzeri, E. Iacob, M. Anderle, C. K. Inoki, T. S. Kuan, P. Jiang, W-1. Wu, J. Vac. Sci. Technol. B 2006, 24 , 1238. 